UT-Komaba/02-01

hep-th/0202079

\title{
Realization of Brane Descent Relations in Effective Theories
}

\author{
Koji Hashimoto* and Satoshi NagaokA ${ }^{\dagger}$ \\ Institute of Physics, University of Tokyo, \\ Komaba, Meguro-ku, Tokyo 153-8902, Japan
}

February, 2002

\begin{abstract}
We examine Sen's descent relations among (non-)BPS D-branes by using low energy effective field theories of $\mathrm{D} p \overline{\mathrm{D}} p$ system. We find that the fluctuation around the kink solution reproduces the low energy matter content on a non$\operatorname{BPS} \mathrm{D}(p-1)$-brane. The effective action for these fluctuation modes turns out to be a generalization of Minahan-Zwiebach model. In addition, it is shown that the fluctuations around the vortex solution consist of massless fields on a BPS D $(p-2)$-brane and they are subject to Dirac-Born-Infeld action. We find the universality that the above results do not refer to particular forms of the effective action.
\end{abstract}

${ }^{*}$ Moved from ITP, USCB. koji@hep1.c.u-tokyo.ac.jp

${ }^{\dagger}$ nagaoka@hep1.c.u-tokyo.ac.jp 


\section{Introduction}

Sen's conjectures on tachyon condensation [1] have contributed immensely to the development of study of non-perturbative features in string theories. The conjectures are basically composed of three parts: (a) The potential height of the tachyon potential exactly cancels the tension of the original unstable D-brane. (b) At the stable true vacuum, the original D-brane disappears and closed string theory is realized. (c) The kink-type tachyon condensations correspond to lower dimensional D-branes and organize "Descent relations" between stable and unstable D-branes (see Fig. 1). These conjectures have been examined from various viewpoints in string/M theory, such as boundary string field theories (BSFT) [2], cubic/vacuum string field theory (C/VSFT), K-theory, and non-commutative solitons. The conjecture (a) was verified [3, 4, 5] in the BSFT, while the conjecture (b) has been challenged by the C/VSFT approach. The conjecture (c) has been supported from the success of the K-theory, and the non-commutative solitons gave a partial evidence also for $(\mathrm{c})$.

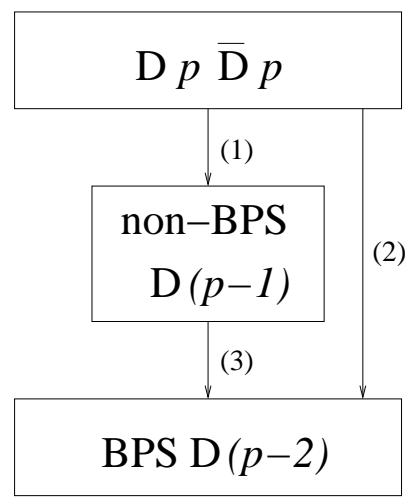

Figure 1: The descent relations for the tachyon condensations. We omit the horizontal arrows which relate type IIA with type IIB, since they only denote the truncation of the fields.

All of the above conjectures are involved with non-perturbative phenomena in string/M theory, thus non-perturbative analyses, such as string field theories treating all the massive modes at once, are necessary. However, we understand that the history of the development of string theory in these six years tells us that the low energy effective theory approach is powerful and promising. This approach is closely related to the BSFT in which one turns on only certain profiles of string excitation modes. Since the study of (b) requires $s \leftrightarrow t$ channel duality, this needs all the massive modes of open string excitation. However, the conjecture (c) is in our reach even by the low energy effective field theory approach, if we appropriately incorporate some of the BSFT results on the exact tachyon potentials.

The field theory models proposed by J. Minahan and B. Zwiebach (we call MZ models) [6, 77, 8] gave a verification of a part of the descent relations (the arrow (3) in Fig. 1 and the descent relations among bosonic branes), at the level of low energy effective field theories (see also [9]). The MZ models include a tachyon field, and were proposed as a toy model of the tachyon condensation. They have kink solutions corresponding to co-dimension one BPS D-branes, and the fluctuations around the solutions are exactly solvable. The resultant fluctuation spectra have equal mass-squared spacing, which resembles to the spectra of the BPS D-brane. In fact, it turned out [3, 7, 5] that the MZ model is actually 
a two-derivative truncation of the BSFT action of a non-BPS D-brane in type II string theories. This consistency provided a further support to the verification by the MZ model.

In this paper, we investigate the descent relations (1) and (2) in Fig. 1, by use of low energy effective field theories. Both arrows have their starting point at the $\mathrm{D} p \overline{\mathrm{D}} p$ system, therefore we use effective actions for the $\mathrm{D} p \overline{\mathrm{D}} p$ which are basically derivative truncation of the BSFT results [10, 11]. The tachyon condensation (1) is the process of creating a co-dimension one defect, therefore we study a kink solution in the $\mathrm{D} p \overline{\mathrm{D}} p$ effective action. The kink represents the non-BPS $\mathrm{D}(p-1)$-brane. Performing the fluctuation analysis around this kink, we obtain an effective action describing the kink, and we show that it coincides with the MZ model action which was the starting point of the arrow (3) in [7, 8]. On the other hand, the process (2) is the creation of a co-dimension two defect, a vortex, which represents a BPS D $(p-2)$-brane. Although Derrick's theorem prevents us from obtaining naive co-dimension two solution, we consider higher derivative terms and obtain a nonsingular vortex solution. We perform the fluctuation spectra and obtain the massless spectra of the BPS brane, and show that those modes obey Dirac-Born-Infeld (DBI) action which is a correct low energy effective action of the BPS brane. One should note that the arrow (2) is different from the naive successive analyses $(1)+(3)$, since the classical solution in (3) is not exactly marginal in (1).

Furthermore, we can show the universality that our results do not depend on the particular form of the action at the starting point. Requiring the properties followed from the BSFT, we write a rather general action and reproduce most of the results above under a few assumptions. We believe that our analysis provides a strong evidence for Sen's conjecture at the effective theory level.

The organization of this paper is as follows. In Sec. 2, after reviewing the MinahanZwiebach model, we show the descent relation from D9D̄9 system to the non-BPS D8brane in Type IIB string theory concerning the tachyon condensation (1) by examining the fluctuations around the kink solution of the D9D̄9 effective action. In Sec. 3, we show the descent relation (2) which is the tachyon condensation from the D9D̄ 9 system to the BPS D7-brane. The universality in each case is shown in the respective sections. We conclude with a summary and discussions in Sec. 4. The appendix is concerning the construction of bound states of D-branes in tachyon condensation.

\section{Kink Solution}

In this section, we derive the Minahan-Zwiebach (MZ) model (which is a low-energy effective description of a non-BPS D8-brane) from a tachyon kink solution in a D9D9 system. This verifies a part of Sen's descent relation, the arrow (1) in Fig. 1, at the level of effective field theories. We focus on the case $p=9$ for the rest of this paper, and generalization to the other dimensions is straightforward. 


\subsection{Review of Minahan-Zwiebach model}

The Minahan-Zwiebach models [6, 0, 8] are low energy effective models which embody the tachyon dynamics for unstable D-branes in (super)string theories. Although the models were first proposed as a toy model capturing desirable properties of string theories, it turned out [3, 7, 5] that one of the models is a derivative truncation of the BSFT action of the non-BPS branes.

Since we shall use some of the techniques developed by Minahan and Zwiebach, in this subsection we review their methods. The "superstring" model which is related to the BSFT is described by the action

$$
S=\mathcal{T} \int d^{9} x e^{-T^{2} / a}\left(1+\left(\partial_{\mu} T\right)^{2}\right) .
$$

This action is the two-derivative truncation of the tachyon action of the BSFT. Here $\mathcal{T}$ is the tension of the non-BPS D8-brane, and the dimensionality of the numerical parameter $a$ is retained by $\alpha^{\prime}$. The equation of motion is solved by a linear tachyon profile $T=x_{8}$ which interpolates two vacua of the theory, $T= \pm \infty$. The center of the kink sits at $x_{8}=0$, which can be viewed from the fact that the perturbative unstable vacuum is at $T=0$. Let us consider the fluctuation $t$ around the kink solution,

$$
T=x_{8}+t(x) .
$$

Substituting this into the action (2.1), we have

$$
S=\mathcal{T} \int d^{9} x e^{-x_{8}^{2} / a}\left(\left(\frac{2 x_{8}^{2}}{a^{2}}-\frac{1}{a}\right) 2 t^{2}-\frac{4}{a} x_{8} t \partial_{8} t+\left(\partial_{\mu} t\right)^{2}\right) .
$$

After a partial integration and the field redefinition

$$
\hat{t} \equiv e^{-x_{8}^{2} / 2 a} t,
$$

we obtain

$$
S=\mathcal{T} \int d^{9} x\left(\left(\partial_{\hat{\mu}} \hat{t}\right)^{2}+\hat{t}\left(-\frac{\partial^{2}}{\partial x_{8}^{2}}+\frac{x_{8}^{2}}{a^{2}}-\frac{1}{a}\right) \hat{t}\right) .
$$

where $\hat{\mu}$ runs over all the directions except for $x_{8}$ which is the direction transverse to the brane. From the above expression, the $x_{8}$ dependence of the fluctuation $\hat{t}_{1}$ obeys a Schrödinger equation of a harmonic oscillator, thus the mass squared for the fluctuation is equally spaced and specified by an integer $n$,

$$
m_{t}^{2}=\frac{2 n}{a}, n \geq 0 .
$$

There is no tachyonic fluctuation, the mass tower starts from a massless state and has the equal spacing. This result is consistent with the identification of this kink as a BPS

\footnotetext{
${ }^{1}$ To obtain the precise mass squared for the tachyon around the perturbative vacuum $T=0$, we have $a=2 \alpha^{\prime}$.
} 
D7-brane. Furthermore, the mass squared of the original tachyon field is $m_{T}^{2}=-\frac{1}{a}$, therefore the spacing of mass levels is twice the value of $\left|m_{T}^{2}\right|$, as is also consistent with string theory.

If we include a gauge field in the original model, then we can get even the gauge modes on the resultant kink BPS D7-brane. Observation in the BSFT tells us that the coupling between the gauge field and the tachyon potential on the non-BPS D8-brane is provided through the Born-Infeld action as [5]

$$
S=\mathcal{T} \int d^{9} x e^{-T^{2} / a} \sqrt{\operatorname{det}\left(1_{\mu \nu}+\sqrt{b} F_{\mu \nu}\right)} .
$$

A sigma-model analysis shows that the parameter $b$ is $\left(2 \pi \alpha^{\prime}\right)^{2}$. We are working in the approximation of the two-derivative truncation,

$$
S=\mathcal{T} \int d^{9} x e^{-T^{2} / a}\left(1+\left(\partial_{\mu} T\right)^{2}+\frac{b}{4} F^{2}\right) .
$$

The classical solution for the action is $T=x_{8}, A_{\mu}=0$. The gauge fluctuation around this solution is subject to the following second order action

$$
S=b \mathcal{T} \int d^{9} x e^{-T^{2} / a}\left(\frac{1}{4} F_{\hat{\mu} \hat{\nu}} F^{\hat{\mu} \hat{\nu}}+\frac{1}{2} \partial_{8} A_{\hat{\mu}} \partial_{8} A^{\hat{\mu}}\right),
$$

where we have chosen the axial gauge condition $A_{8}=0$. After the field redefinition $\hat{A}_{\hat{\mu}} \equiv e^{-x_{8}^{2} / 2 a} \hat{A}_{\hat{\mu}}$, the action (2.9) is rewritten as

$$
S=b \mathcal{T} \int d^{9} x\left[\frac{1}{4} \hat{F}_{\hat{\mu} \hat{\nu}} \hat{F}^{\hat{\mu} \hat{\nu}}+\frac{1}{2} \hat{A}_{\hat{\mu}}\left(-\frac{\partial^{2}}{\partial x_{8}^{2}}+\frac{x_{8}^{2}}{a^{2}}-\frac{1}{a}\right) \hat{A}_{\hat{\mu}}\right] .
$$

Therefore, the mass spectrum of the gauge fluctuation starts from massless gauge modes and is equally spaced,

$$
m_{A_{\hat{\mu}}}^{2}=\frac{2 n}{a}, \quad n \geq 0 .
$$

This result is also consistent with the expectation from the spectra on the BPS D7-brane.

The Minahan-Zwiebach "superstring" model provides a suitable example realizing various aspects of the tachyon condensation on non-BPS branes, in particular a part of the descent relations. If The background consistency with the BSFT ensures this success, and we employ this philosophy to check the other part of the descent relations in the rest of this paper.

\footnotetext{
${ }^{2}$ Furthermore, it is known that the massless fields obtained here generally obeys the dimensionally reduced Born-Infeld action which is an effective action of a D7-brane [12].
} 


\subsection{Kink solution in brane antibrane system}

The other important part in Sen's descent relations is the relation between the D9D̄9 and a non-BPS D8-brane. The latter is conjectured to be a kink solution of the former. In order to show this conjecture at the effective theory level, first we follow the procedures of the analysis reviewed in the previous subsection.

The effective action of the D9Dי 9 system in the two derivative truncation is written as 10, 11

$$
S=2 T_{\mathrm{D} 9} \int d^{10} x e^{-|T|^{2} / a}\left(1+\left|D_{\mu}^{(-)} T\right|^{2}+\frac{1}{4} b\left(F_{\mu \nu}^{(-)}\right)^{2}+\frac{1}{4} b\left(F_{\mu \nu}^{(+)}\right)^{2}\right),
$$

where $T \equiv T_{1}+i T_{2}$ is a complex tachyon charged under the gauge field $A_{\mu}^{(-)}$,

$$
D_{\mu}^{(-)} T=\partial_{\mu} T-i A_{\mu}^{(-)} T \text {. }
$$

The fields $A_{\mu}^{( \pm)}$are the linear combinations of the gauge fields on the brane and the antibrane,

$$
A_{\mu}^{( \pm)}=A_{\mu}^{(1)} \pm A_{\mu}^{(2)}
$$

and $a, b$ are dimensionful numerical constant which can be determined by the BSFT analysis, string sigma model calculation, or string scattering amplitude. [?

Classical solution

The equations of motion for this action are

$$
\begin{aligned}
& -\frac{1}{a}\left[\bar{T}\left(1+\frac{b}{4}\left(F_{\mu \nu}^{(-)}\right)^{2}+\frac{b}{4}\left(F_{\mu \nu}^{(+)}\right)^{2}\right)-T\left(D_{\mu} \bar{T}\right)^{2}\right]-D_{\mu} D_{\mu} \bar{T}=0, \\
& \partial_{\nu}\left(e^{-|T|^{2} / a} F_{\mu \nu}^{(+)}\right)=0, \\
& i e^{-|T|^{2} / a}\left(T D_{\mu} \bar{T}-\bar{T} D_{\mu} T\right)+b \partial_{\nu}\left(e^{-|T|^{2} / a} F_{\mu \nu}^{(-)}\right)=0 .
\end{aligned}
$$

Note that putting $T_{2}=A^{(-)}=0$ makes the action and equations of motion reduce to those of the MZ model. In particular, the third equation (2.17) which came from the differentiation with respect to $A^{(-)}$is trivially satisfied by this condition. Therefore, the kink solution of the MZ model is inherited to our situation. The desired solution is

$$
T=x_{9}, \quad A_{\mu}^{( \pm)}=0 .
$$

$\underline{\text { Fluctuation analysis }}$

Let us consider the fluctuation around this kink solution. As is obvious, the fluctuation spectra for the fields $T_{1}$ and $A_{\mu}^{(+)}$are precisely the same as in the previous subsection, thus we obtain

$$
m_{t_{1}}^{2}=\frac{2 n_{1}}{a}, \quad m_{A_{\hat{\mu}}^{(+)}}^{2}=\frac{2 n_{2}}{a} \quad \text { where } \quad n_{i} \geq 0, \quad i=1,2 .
$$

\footnotetext{
${ }^{3}$ To be precise, here we have $a=2 \alpha^{\prime}$ and $b=\left(2 \pi \alpha^{\prime}\right)^{2}$, as mentioned in the previous subsection. Note that the value of $a$ depends on the renormalization scheme in the BSFT and the sigma model approach [13.
} 
Here we denote the fluctuation of $T_{1}$ as $t_{1}$. The lowest modes of these fluctuations are zero modes, and especially the $t_{1}$ zero mode corresponds to the scalar field on the nonBPS D8-brane, representing the transverse displacement of the brane. This must have appeared by the Nambu-Goldstone theorem, since the translational invariance along $x_{9}$ direction is broken by the kink solution.

Another notable point about the zero modes is the $x_{9}$ dependence. Since we solved the harmonic oscillator problem for $\hat{t}_{1}$ and $\hat{A}_{\mu}^{(+)}$, the ground state's wave functions are gaussian:

$$
\left[x_{9} \text { part of } \hat{t}_{1}\left(x_{0}, x_{1}, \cdots, x_{9}\right)\right]=e^{-\frac{x_{9}^{2}}{a}} .
$$

Therefore, turning back to the field redefinition (2.4), we can show that the zero modes of $t_{1}, A_{\mu}^{(+)}$are independent of $x_{9}$. This kind of the property of the lowest fluctuation was observed first in the bosonic D-brane system [9].

The fluctuation analysis for the other fields $T_{2}$ and $A_{\mu}^{(-)}$can be performed in the same manner.f First, we consider the fluctuation of $T_{2}$ around the classical solution which is written as

$$
T=x_{9}+i t_{2}(x), A_{\mu}^{( \pm)}=0 .
$$

Substituting this into the action (2.12) and picking up the second order terms, we obtain

$$
S_{t_{2}}=2 T_{\mathrm{D} 9} \int d^{10} x e^{-\frac{x_{9}^{2}}{a}}\left(-\frac{2}{a} t_{2}^{2}+\left(\partial_{\mu} t_{2}\right)^{2}\right) .
$$

After the same redefinition $\hat{t}_{2}=e^{-\frac{x_{9}^{2}}{2 a}} t_{2}$, the fluctuation action is written as

$$
S=2 T_{\mathrm{D} 9} \int d^{10} x\left(\left(\frac{x_{9}^{2}}{a^{2}}-\frac{3}{a}\right) \hat{t}_{2}^{2}+\left(\partial_{\mu} \hat{t}_{2}\right)^{2}\right) .
$$

Therefore the series of the mass squared begins with the negative mass squared, which is expected to exist as a tachyonic mode of the non-BPS D8-brane,

$$
m_{t_{2}}^{2}=\frac{2(n-1)}{a}, \quad n \geq 0
$$

The mass squared spacing is the same as that of $t_{1}$ fluctuations. Again, the lowest mode is independent of $x_{9}$.

Since there should be a single massless gauge field on the resultant non-BPS D8-brane, the fluctuation of another gauge field $A_{\mu}^{(-)}$must be massive. We shall see this below. The fluctuation we consider here is

$$
T=x_{9}, A_{\mu}^{(+)}=0, A_{\mu}^{(-)}=A_{\mu}^{(-)}(x) .
$$

\footnotetext{
${ }^{4}$ We treat fluctuations of the fields one by one separately, since generically it is difficult to diagonalize all of the fluctuations. We shall discuss this point in the final section.
} 
Following the same procedures with the field redefinition $\hat{A}_{\mu}^{(-)}=e^{-\frac{x_{9}^{2}}{2 a}} A_{\mu}^{(-)}$, we have

$$
S=2 T_{\mathrm{D} 9} \int d^{10} x\left(\frac{b}{4}\left(\hat{F}_{\hat{\mu} \hat{\nu}}^{(-)}\right)^{2}+\frac{b}{2} \hat{A}_{\hat{\mu}}^{(-)}\left[-\partial_{9}^{2}+\left(\frac{2}{b}+\frac{1}{a^{2}}\right) x_{9}^{2}-\frac{1}{a}\right] \hat{A}_{\hat{\mu}}^{(-)}\right),
$$

where the indices $\hat{\mu}, \hat{\nu}$ run over $0,1, \cdots, 8$, and the gauge fixing condition $\partial_{\hat{\mu}} \hat{A}_{\hat{\mu}}^{(-)}=0$ has been used. The mass squared is given as

$$
m_{A_{\hat{\mu}}^{(-)}}^{2}=2 n \sqrt{\frac{2}{b}+\frac{1}{a^{2}}}+\sqrt{\frac{2}{b}+\frac{1}{a^{2}}}-\frac{1}{a}, \quad n \geq 0 .
$$

The lowest mode of these fluctuations is massive, as expected. It does not appear in the low energy effective theory of the kink.

We have seen that $A^{(+)}$still has a massless mode while $A^{(-)}$becomes massive. One of the physical explanation is as follows. The tachyon field couples only to $A^{(-)}$, thus if the tachyon condenses, the gauge field $A^{(-)}$is Higgsed and becomes massive. On the other hand, $T$ is neutral under $A^{(+)}$, thus $A^{(+)}$stays massless after the tachyon condensation. One can see this in another way. The gauge field $A^{(+)}$is responsible for the lowerdimensional D-brane charges, as seen in App. A as an example. Therefore $A^{(+)}$should remain massless on the kink (or also on the vortex as we shall see in the next section).

\section{$\underline{\text { Effective action }}$}

In the end, we summarize this subsection by writing the total effective action obtained from the above fluctuation analysis. The gauge field $A^{(-)}$does not appear in the low energy region. We substitute the lowest fluctuation modes into the action (2.12) and obtain

$$
\begin{aligned}
S_{\mathrm{tot}} & =2 T_{\mathrm{D} 9} \int d^{10} x e^{-\left(x_{9}+t_{1}\right)^{2} / a-t_{2}^{2} / a}\left(2+\left(\partial_{\hat{\mu}} t_{1}\right)^{2}+\left(\partial_{\hat{\mu}} t_{2}\right)^{2}+\frac{b}{4}\left(F_{\hat{\mu} \hat{\nu}}^{(+)}\right)^{2}\right) \\
& =2 T_{\mathrm{D} 9} \int d^{9} x e^{-t_{2}^{2} / a}\left(2+\left(\partial_{\hat{\mu}} t_{1}\right)^{2}+\left(\partial_{\hat{\mu}} t_{2}\right)^{2}+\frac{b}{4}\left(F_{\hat{\mu} \hat{\nu}}^{(+)}\right)^{2}\right) \int d\left(x_{9}+t_{1}\left(x_{\hat{\mu}}\right)\right) e^{-\left(x_{9}+t_{1}\right)^{2} / a} \\
& =2 T_{\mathrm{D} 9} \sqrt{a \pi} \int d^{9} x e^{-t_{2}^{2} / a}\left(2+\left(\partial_{\hat{\mu}} t_{2}\right)^{2}+\left(\partial_{\hat{\mu}} t_{1}\right)^{2}+\frac{b}{4}\left(F_{\hat{\mu} \hat{\nu}}^{(+)}\right)^{2}\right)
\end{aligned}
$$

In the second equality, we have used the fact that $t_{1}, t_{2}$, and $A_{\mu}^{(+)}$are independent of $x_{9}$. The third equality is obtained by the integration over the transverse direction. Due to the gaussian factor, we can say that the defect described by the action (2.28) is located at $x_{9}=-t_{1}\left(x_{\hat{\mu}}\right)$. This action (2.28) is precisely identical with the effective action of a non-BPS D8-brane. The difference from the action of the MZ model (2.1) is that now it is generalized to incorporate the transverse scalar field on the non-BPS brane. The way it is included in is just the same as the two-derivative truncation of the BSFT action for the non-BPS D8-brane.

Thus, we have shown the decent relation from D9D̄9 system to the non-BPS D8-brane by directly examining the fluctuations around the kink solution of the D9D̄9 effective action.

\footnotetext{
${ }^{5}$ However, our tachyon condensation is a linear profile, hence a naive mass of the Higgsed gauge field $A^{(-)}$vanishes at $x^{9}=0$. This is against our expectation and too naive, thus we have needed the precise fluctuation analysis (2.27).
} 


\subsection{General action and universality of the results}

Although the results obtained in the previous subsection are desirable, they may depend on the choice of the effective theories. In this subsection, we show that the results of the previous subsection are in fact quite general, in the sense that the results do not depend on the specific form of the Lagrangian. If we remember that the preferable properties of the MZ model are intrinsically due to its relation to the BSFT, the general Lagrangian should be constrained by some results of the BSFT. Here we respect the properties of the BSFT action derived for linear tachyon profiles. Since in the BSFT it is difficult to treat $A^{(-)}$generically, we neglect this gauge field in this general approach.

The symmetries which the model should have are (i) Lorentz invariance, (ii) gauge invariance with respect to $A^{(+)}$, and (iii) the global gauge invariance associated originally with $A^{(-)}$,

$$
T \mapsto e^{i \theta} T .
$$

Employing the BSFT and the sigma-model result, the coupling between the gauge field $A^{(+)}$and the tachyon is determined (under the assumption that we consider the constant gauge field strength and neglect the tachyon higher derivatives $\partial \partial T$ ) as

$$
S=2 T_{\mathrm{D} 9} \int d^{10} x e^{-|T|^{2} / a} \sqrt{\operatorname{det}(1+\sqrt{b} F)} f(X, Y) .
$$

In the above expression the invariants $X$ and $Y$ are defined as

$$
X \equiv G^{\mu \nu} \partial_{\mu} T \partial_{\nu} \bar{T}, \quad Y \equiv\left|G^{\mu \nu} \partial_{\mu} T \partial_{\nu} T\right|^{2},
$$

and the metric $G$ is in the well-known form,

$$
G^{\mu \nu}=\left(\frac{1}{1-b F^{2}}\right)^{\mu \nu} .
$$

We omit the upper case $(+)$ here and in the rest of this subsection.

\section{Classical solution}

In order for the fluctuation analysis to be performed, we require that a classical solution of the above system should be a linear profile of the tachyon:

$$
T=q x_{9}, \quad A_{\mu}=0 .
$$

Then the equations of motion become a constraint on the unknown function $f(X, Y)$ :

$$
\left[f-2 q^{2} \frac{\delta f}{\delta X}-4 q^{4} \frac{\delta f}{\delta Y}\right]_{X=q^{2}, Y=q^{4}}=0 .
$$

Fluctuation analysis

First let us carry out the $T_{1}$ fluctuation. Substituting $T=q x_{9}+t_{1}(x)$ and $A_{\mu}=0$ into the action (2.30) and picking up the quadratic terms, we obtain

$$
S=2 T_{\mathrm{D} 9} \int d^{10} x e^{-q^{2} x_{9}^{2} / a}\left(C_{1}\left(\partial_{8} t_{1}\right)^{2}+\frac{f}{2 q^{2}}\left(\partial_{\hat{\mu}} t_{1}\right)^{2}\right),
$$


where

$$
C_{1} \equiv\left[\frac{\delta f}{\delta X}+6 q^{2} \frac{\delta f}{\delta Y}+2 q^{2} \frac{\delta^{2} f}{\delta X^{2}}+8 q^{6} \frac{\delta^{2} f}{\delta Y^{2}}+8 q^{4} \frac{\delta^{2} f}{\delta X \delta Y}\right]_{X=q^{2}, Y=q^{4}}
$$

Note that we use the equation of motion (2.34) here. Then the mass squared is

$$
m_{t_{1}}^{2}=\frac{4 q^{4} C_{1}}{a f\left(q^{2}, q^{4}\right)} n, \quad n \geq 0
$$

therefore there is a massless mode which corresponds to the transverse scalar field on the non-BPS D8-brane, that is the same as in the derivative truncated case.

It is straightforward to check that this is also the case for the $T_{2}$ fluctuation. Substituting $T=q x_{9}+i t_{2}(x), A_{\mu}=0$ into the action, we have

$$
S=2 T_{\mathrm{D} 9} \int d^{10} x\left(\frac{f\left(q^{2}, q^{4}\right)}{2 q^{2}} \hat{t}_{2}\left(-\partial_{9}^{2}+\frac{q^{4}}{a^{2}} x_{9}^{2}-\frac{3 q^{2}}{a}\right) \hat{t}_{2}+\left(\partial_{\mu} \hat{t}_{2}\right)^{2}\left[\frac{\delta f}{\delta X}-2 q^{2} \frac{\delta f}{\delta Y}\right]_{\substack{X=q^{2} \\ Y=q^{4}}}\right) .
$$

The mass squared is

$$
m_{t_{2}}^{2}=(n-1)\left[\frac{f}{a}\left(\frac{\delta f}{\delta X}-2 q^{2} \frac{\delta f}{\delta Y}\right)^{-1}\right]_{\substack{X=q^{2} \\ Y=q^{4}}}, \quad n \geq 0
$$

thus there is a tachyonic mode.

However, the result for the gauge fluctuation is a little bit different. The analysis for the gauge fluctuations gives the following quadratic action,

$$
S=2 T_{\mathrm{D} 9} \frac{b f\left(q^{2}, q^{4}\right)}{4} \int d^{10} x e^{-\frac{q^{2}}{a} x_{9}^{2}} F_{\hat{\mu} \hat{\nu}}^{2} .
$$

The remarkable point is the vanishing of the $A_{\hat{\mu}}^{2}$ terms, therefore we have no massive tower, as opposed to the case of the two-derivative truncation. We have only the massless gauge mode localized on the kink solution. Note that, as for the gauge field, our general analysis does not include the action (2.12) of the previous subsection, because we have another contribution for $F^{2}$ from the metric $G^{\mu \nu}$.

The lowest modes for the tachyon fluctuations $t_{1}$ and $t_{2}$ are independent of $x_{9}$, as before. On the other hand, as seen above, the gauge fluctuation has no tower and hence no information on its $x_{9}$ dependence, thus we cannot include the gauge fluctuation in obtaining the effective action of the kink in the same manner as (2.28). However, in the expression (2.39) the gaussian factor in front of the field strength behave as a spacedependent dielectric constant [14], thus the massless gauge field is localized on the kink. We conclude that we have obtained the precise field content on the non-BPS D8-brane by the fluctuation analysis in the general action (2.30). 


\section{Vortex Solution}

As mentioned in the introduction, how the stable BPS branes appear in the tachyon condensation of the brane antibrane system is the basis of the K-theory argument. After the development of the techniques to deal with the tachyon condensation such as the BSFT, we can now closely look at the nucleation of the lower dimensional BPS D-branes after the tachyon condenses. In this section, we focus on the creation of a BPS D7brane as a vortex solution, [ from the tachyon condensation of the D9D̄9 system. After constructing the solution, we perform the fluctuation analysis and then see if the precise worldvolume field content appears on the vortex D7-brane. We will see that the general analysis independent of the form of the action, studied in Sec. 2.3, can be applied also here. Finally we derive the DBI action as an effective field theory of the fluctuation modes.

\subsection{Vortex solution for the tachyon field}

Since the inclusion of the gauge fields is accompanied by some complication which we shall see in Sec. 3.3, first we consider a simple situation where we treat only the complex tachyon field. The action in the two derivative truncation is written as

$$
S=2 T_{\mathrm{D} 9} \int d^{10} x e^{-|T|^{2} / a}\left(1+\left|\partial_{\mu} T\right|^{2}\right) .
$$

Although the D7-brane should be realized as a co-dimension two vortex solution of the tachyon system, the well-known Derrick's theorem [15] prohibit us from proceeding. Let us recall how Derrick's theorem applies to our situation with the action (3.1). Make the tachyon profile to depend only on two coordinates among the worldvolume directions, and suppose that we have a finite energy solution $T=T_{\text {sol }}$. Then, the rescaling $T_{\text {sol }}(x) \mapsto$ $T^{\prime}=T_{\text {sol }}(\lambda x)$ changes the energy (action) to

$$
\begin{aligned}
S^{\prime} & =2 T_{\mathrm{D} 9} V_{\mathrm{D} 7} \int d^{2} x e^{-\left|T^{\prime}\right|^{2} / a}\left(1+\left|\partial_{\mu} T^{\prime}\right|^{2}\right) \\
& =2 T_{\mathrm{D} 9} V_{\mathrm{D} 7} \int d^{2} x^{\prime} e^{-\left|T_{\mathrm{sol}}\left(x^{\prime}\right)\right|^{2} / a}\left(\lambda^{2}+\left|\partial_{\mu}^{\prime} T_{\mathrm{sol}}\left(x^{\prime}\right)\right|^{2}\right),
\end{aligned}
$$

where $x^{\prime} \equiv \lambda x$. Since $T_{\text {sol }}$ with $\lambda=1$ should minimizes the energy $\frac{d}{d \lambda} E\left(T_{\text {sol }}\right)=0$, we obtain

$$
\int d^{2} x e^{-\left|T_{\mathrm{sol}}\right|^{2} / a}=0,
$$

which tells us that the tachyon solution should sit at the potential minimum everywhere, $\left|T_{\text {sol }}\right|=\infty$. This shows that there exists no vortex solution as far as we employ the action (3.1). To avoid this no-go theorem, we have to include terms supplying negative powers in $\lambda$ to stabilize the system against the rescaling, such as tachyon higher derivative terms

\footnotetext{
${ }^{6}$ We use the term "vortex" in a broad sense, not only for the usual gauge vortex. Following Sen's original usage, we specifically call the tachyon configuration (3.8) "vortex".
} 
or gauge field kinetic terms. In this and the next subsections, we adopt the first approach. For the latter approach, see [16]. 1]

Let us consider an action of the four derivative truncation. The form of the additional terms respects the BSFT results[10, 11],

$$
S=2 T_{\mathrm{D} 9} \int d^{10} x e^{-|T|^{2} / a}\left(1+\left|\partial_{\mu} T\right|^{2}+p_{1}\left|\partial_{\mu} T\right|^{4}+p_{2}\left(\partial_{\mu} T\right)^{2}\left(\partial_{\nu} \bar{T}\right)^{2}\right) .
$$

New coefficients $p_{1}$ and $p_{2}$ are some numerical constant. The origin of these higher derivative terms is as follows. The BSFT action obtained in 10, 11 for the linear tachyon profile is given by

$$
S=\int d^{10} x e^{-|T|^{2}} \Pi_{i=1,2} F\left(u_{i}^{2}\right)
$$

where $T=u_{1} x_{8}+i u_{2} x_{9}$ and $F(x)=\frac{4^{x} x \Gamma(x)}{2 \Gamma(x)}$. We omit numerical coefficients and $\alpha^{\prime}$ for simplicity. Performing Taylor-expansion for this action around $u=0$ and restoring the Lorentz invariance and the global gauge invariance (2.29), we obtain the action (3.4).

We can rephrase this in another way. Suppose that the action can be factorized as

$$
S=2 T_{\mathrm{D} 9} \int d^{10} x e^{-|T|^{2} / a} f(\partial T),
$$

then the general terms of the order $(\partial T)^{4}$ in $f$, which are Lorentz invariant and global gauge invariant, are only the ones in the action (3.4).

\section{Classical solution}

Assuming that the tachyon solution is linear, the equations of motion for this action (3.4) reduce to

$$
\bar{T}\left[1-p_{1}\left|\partial_{\mu} T\right|^{2}-p_{2}\left(\partial_{\mu} T\right)^{2}\left(\partial_{\nu} \bar{T}\right)^{2}\right]-T\left(\partial_{\mu} \bar{T}\right)^{2}\left[1+2\left(p_{1}+p_{2}\right)\left|\partial_{\nu} T\right|^{2}\right]=0 .
$$

A linear vortex solution is found as

$$
T=q\left(x_{8}+i x_{9}\right),
$$

where the real parameter $q$ satisfies $4 q^{4} p_{1}=1$. The true vacuum of the tachyon potential $e^{-|T|^{2} / a}$ is $S^{1}$ at the infinity of the tachyon configuration space, $|T|=\infty$. We deal only with the co-dimension two solitons, hence the solitons are categorized by the homotopy group $\pi_{1}\left(S^{1}\right)=\mathbb{Z}$ characterizing the winding number of the solitons. The above solution (3.8) has a unit charge in this sense.

The reason why we have assumed the solution to be linear is that linear solutions with the winding number one are directly related to the Ramond-Ramond charge of the BPS D7-brane, through the Chern-Simons (CS) term of the D9D̄9 effective action computed in [10, 11]. The relevant CS term is given by

$$
S_{\mathrm{CS}}=2 T_{\mathrm{D} 9} \int C \wedge \operatorname{Tr} \sigma_{3} \exp \left(\begin{array}{cc}
-|T|^{2} & d \bar{T} \\
d T & -|T|^{2}
\end{array}\right),
$$

\footnotetext{
${ }^{7}$ The action used in 16 is slightly different from the BSFT results.
} 
where we set the gauge field to vanish, and omit numerical parameters for simplicity. Substituting the solution (3.8) into the above expression (3.9), we can obtain the D7brane charge,

$$
S_{\mathrm{CS}}=2 T_{\mathrm{D} 9} \int e^{-|T|^{2}} C^{(8)} \wedge d T \wedge d \bar{T} \sim \int_{V_{\mathrm{D} 7}} C^{(8)}
$$

Fluctuation analysis

Since the solution has a symmetry of interchanging $x_{8}$ and $x_{9}$ simultaneously with the exchange of $T_{1}$ and $T_{2}$, it is sufficient to consider the fluctuation of $T_{1}$ around the vortex solution (3.8), $T=q\left(x_{8}+i x_{9}\right)+t_{1}(x)$. Substituting this into (3.4), we have the action

$$
\begin{gathered}
S=2 T_{\mathrm{D} 9} \int d^{10} x e^{-\frac{q^{2}\left(x_{8}^{2}+x_{9}^{2}\right)}{a}}\left[2\left(\frac{2 q^{2}}{a^{2}} x_{8}^{2}-\frac{1}{a}\right)\left(1+q^{2}\right) t_{1}^{2}-\frac{4\left(1+q^{2}\right)}{a} x_{8} t_{1} \partial_{8} t_{1}\right. \\
\left.+\frac{1+q^{2}}{q^{2}}\left(\partial_{\hat{\mu}} t_{1}\right)^{2}+\frac{1}{q^{2}}\left(\partial_{8} t_{1}\right)^{2}+4 p_{2} q^{2}\left(\left(\partial_{8} t_{1}\right)^{2}+\left(\partial_{9} t_{1}\right)^{2}\right)\right]
\end{gathered}
$$

where we have extracted only the terms quadratic in $t_{1}$. After performing the field redefinition

$$
\hat{t}_{1}=t_{1} \exp \left[-\frac{q^{2}\left(x_{8}^{2}+x_{9}^{2}\right)}{2 a}\right],
$$

we can eliminate the exponential factor in the action as

$$
\begin{aligned}
S= & 2 T_{\mathrm{D} 9} \int d^{10} x\left(\left(2+q^{2}+4 p_{2} q^{4}\right)\left(\frac{q^{2}}{a^{2}} x_{8}^{2} \hat{t}_{1}^{2}+\frac{1}{q^{2}}\left(\partial_{8} \hat{t}_{1}\right)^{2}-\frac{1}{a} \hat{t}_{1}^{2}\right)\right. \\
& \left.+\left(1+q^{2}+4 p_{2} q^{4}\right)\left(\frac{q^{2}}{a^{2}} x_{9}^{2} \hat{t}_{1}^{2}+\frac{1}{q^{2}}\left(\partial_{9} \hat{t}_{1}\right)^{2}-\frac{1}{a} \hat{t}_{1}^{2}\right)+\frac{1+q^{2}}{q^{2}}\left(\partial_{\hat{\mu}} \hat{t}_{1}\right)^{2}\right)
\end{aligned}
$$

where $\hat{\mu}$ denotes the directions along the worldvolume of the defect, $0,1, \cdots, 7$. Solving the two-dimensional harmonic oscillator problem, the mass squared is found as

$$
m_{t_{1}}^{2}=\frac{2 q^{2}}{a\left(1+q^{2}\right)}\left(\left(2+q^{2}+4 p_{2} q^{4}\right) n_{1}+\left(1+q^{2}+4 p_{2} q^{4}\right) n_{2}\right), \quad n_{1}, n_{2} \geq 0
$$

The lowest mode for the fluctuations is massless and corresponds to the Nambu-Goldstone (NG) boson associated with the breaking of the translational invariance along $x_{8}$. This mode is in fact the transverse scalar field on the BPS D7-brane. When we replace $t_{1}$ by $t_{2}$ which is the fluctuation for $T_{2}$, from the symmetry mentioned above, we obtain the same result and get the transverse NG mode for the $x_{9}$ direction. This is also expected to exist on the D7-brane. 


\subsection{Vortex solution in the brane antibrane system with gauge fields}

Let us proceed to include the gauge fields on the D9D̄9 system. First we treat the gauge field $A_{\mu}^{(+)}$. We employ a similar gauge coupling as the MZ model, then the total action is written as

$$
S=2 T_{\mathrm{D} 9} \int d^{10} x e^{-|T|^{2} / a}\left(1+\left|\partial_{\mu} T\right|^{2}+\frac{b}{4}\left(F^{(+)}\right)^{2}+p_{1}\left(\left|\partial_{\mu} T\right|^{2}\right)^{2}+p_{2}\left(\partial_{\mu} T\right)^{2}\left(\partial_{\nu} \bar{T}\right)^{2}\right)(3
$$

A classical solution naively extended is

$$
T=q\left(x_{8}+i x_{9}\right), \quad A_{\mu}^{(+)}=0,
$$

and we consider the fluctuation $A_{\mu}^{(+)}=A_{\mu}(x)$ around this solution. Substituting these into (3.15), we obtain

$$
\begin{gathered}
S=\quad 2 T_{\mathrm{D} 9} \int d^{10} x\left(\hat{A}_{\hat{\mu}}\left[-\partial_{8}^{2}-\partial_{9}^{2}+\frac{q^{4}}{a^{2}}\left(x_{8}^{2}+x_{9}^{2}\right)-\frac{2 q^{2}}{a}\right] \hat{A}_{\hat{\mu}}+\frac{1}{2} \hat{F}_{\hat{\mu} \hat{\nu}}^{2}\right. \\
+\hat{A}_{8}\left[-\partial_{\hat{\mu}}^{2}-\partial_{9}+\frac{q^{4}}{a^{2}} x_{9}^{2}-\frac{q^{2}}{a}\right] \hat{A}_{8}+\hat{A}_{9}\left[-\partial_{\hat{\mu}}^{2}-\partial_{8}^{2}+\frac{q^{4}}{a^{2}} x_{8}^{2}-\frac{q^{2}}{a}\right] \hat{A}_{9} \\
+\frac{2 q^{2}}{a} x_{8} \hat{A}_{8}\left[\partial_{\hat{\mu}} \hat{A}_{\hat{\mu}}+\partial_{9} A_{9}\right]+\frac{2 q^{2}}{a} x_{9} \hat{A}_{9}\left[\partial_{\hat{\mu}} \hat{A}_{\hat{\mu}}+\partial_{8} A_{8}\right]-\frac{2 q^{4}}{a^{2}} x_{8} x_{9} \hat{A}_{8} \hat{A}_{9} \\
\left.-2\left(\partial_{8} \hat{A}_{8} \partial_{9} \hat{A}_{9}+\partial_{8} \hat{A}_{8} \partial_{\hat{\mu}} \hat{A}_{\hat{\mu}}+\partial_{9} \hat{A}_{9} \partial_{\hat{\mu}} \hat{A}_{\hat{\mu}}\right)\right)
\end{gathered}
$$

where we have already redefined the gauge field as $\hat{A}_{\mu} \equiv A_{\mu} \exp \left(-q^{2}\left(x_{8}^{2}+x_{9}^{2}\right) / 2 a\right)$. This action has cross terms between $\hat{A}_{\hat{\mu}}$ and $\hat{A}_{8,9}$, hence it is difficult to diagonalize this action. But if we choose the gauge condition $\partial_{\hat{\mu}} \hat{A}_{\hat{\mu}}=0$, the $\hat{A}_{\hat{\mu}}$ part can be separated from the rest $\hat{A}_{8.9}$. In the following, we shall consider only the separated $\hat{A}_{\hat{\mu}}$ part which is the first line of the above action. The mass squared is easily obtained as

$$
m_{A_{\hat{\mu}}^{(+)}}^{2}=\frac{2 q^{2} n}{a}, \quad n \geq 0 .
$$

Thus, $A_{\hat{\mu}}^{(+)}$has a single massless gauge field on the vortex, which is one of the correct massless content on the BPS D7-brane.

Second, we move to the analysis of the fluctuations for $A_{\mu}^{(-)}$. The action we should consider here is the following:

$$
\begin{array}{r}
S=2 T_{\mathrm{D} 9} \int d^{10} x e^{-|T|^{2} / a}\left(1+D_{\mu} T D^{\mu} \bar{T}+\frac{b}{4}\left(F^{(-)}\right)^{2}+i c F_{\mu \nu}^{(-)} D_{\mu} T D_{\nu} \bar{T}\right. \\
\left.+p_{1}\left(D_{\mu} T D^{\mu} \bar{T}\right)^{2}+p_{2}\left(D_{\mu} T\right)^{2}\left(D_{\mu} \bar{T}\right)^{2}\right) .
\end{array}
$$

Here $c$ is another numerical parameter. The derivative is now replaced by the covariant derivative, because $T$ is charged under the gauge field $A_{\mu}^{(-)}$. We choose the following gauge choice

$$
x_{8} A_{9}-x_{9} A_{8}=0 .
$$


Under this gauge choice, the equations of motion can be solved by

$$
T=q\left(x_{8}+i x_{9}\right), \quad A_{\mu}^{(-)}=0,
$$

with $4 q^{4} p_{1}=1$. Considering the $A_{\mu}^{(-)}$gauge fluctuations around the vortex solution, we obtain the action

$$
\begin{array}{r}
S=2 T_{\mathrm{D} 9} \int d^{10} x e^{-\frac{q^{2}}{a}\left(x_{8}^{2}+x_{9}^{2}\right)}\left(q^{2}\left(x_{8}+x_{9}^{2}\right) A_{\mu}^{2}+\left(x_{8}^{2}+x_{9}^{2}\right)^{2} A_{\mu}^{2}+4 q^{4} p_{2}\left(x_{8}^{2}+x_{9}^{2}\right)\left(A_{8}^{2}+A_{9}^{2}\right)\right. \\
\left.+\frac{b}{4} F_{\mu \nu}^{2}+2 c q^{2}\left[F_{\hat{\mu} 8} x_{8} A_{\hat{\mu}}+F_{\hat{\mu} 9} x_{9} A_{\hat{\mu}}\right]+(\text { cross terms })\right)
\end{array}
$$

After the field redefinition $\hat{A}_{\mu} \equiv A_{\mu} \exp \left(-q^{2}\left(x_{8}^{2}+x_{9}^{2}\right) / 2 a\right)$, we have

$$
\begin{aligned}
S=2 T_{\mathrm{D} 9} \int d^{10} x( & \hat{A}_{\hat{\mu}}\left[\frac{b}{2}\left(-\partial_{8}^{2}-\partial_{9}^{2}+\frac{q^{4}}{a^{2}}\left(x_{8}^{2}+x_{9}^{2}\right)-\frac{2 q^{2}}{a}\right)\right. \\
& \left.+2 c q^{2}\left(-\frac{q^{2}}{a}\left(x_{8}^{2}+x_{9}^{2}\right)+1\right)+\left(1+q^{2}\right)\left(x_{8}^{2}+x_{9}^{2}\right)\right] \hat{A}_{\hat{\mu}} \\
& \left.+\frac{b}{4} \hat{F}_{\hat{\mu} \hat{\nu}}^{2}+\left(A_{8}, A_{9} \text { terms }\right)\right) .
\end{aligned}
$$

Since we have already used the gauge freedom, we cannot eliminate the cross terms unfortunately. In the following analysis, we simply put $\hat{A}_{8}=\hat{A}_{9}=0$ for our convenience. Then the mass squared for the $\hat{A}_{\hat{\mu}}$ is

$$
m_{A_{\hat{\mu}}^{(-)}}^{2}=(2 n+2) \sqrt{\frac{2}{b}\left(1+q^{2}+\frac{b q^{4}}{2 a^{2}}-c \frac{2 q^{4}}{a}\right)}-\frac{2 q^{2}}{a}+c \frac{4 q^{2}}{b}, \quad n \geq 0 .
$$

Whether the lowest mass squared is positive, negative or zero depends on the numerical value of, in particular, $c$. If we set $c=0$, the lowest mass squared becomes positive, as desired. $p^{p}$

We have seen that the parameters in the action are sensitive to the resultant fluctuation spectra. In the following, we present another approach which might be better than the approach above, in the sense that we need no information on $c$.

The sensitive terms are higher derivatives, thus we stick to the two-derivative truncation. Noting that Derrick's theorem does not mention about anything on the existence of a singular solution, we introduce a regularization

$$
S=2 T_{\mathrm{D} 9} \int d^{10} x e^{-|T|^{2} / a}\left(1+\left(D_{\mu} T D^{\mu} \bar{T}\right)^{1+\epsilon}+\frac{b}{4} F_{\mu \nu}^{2}\right) .
$$

\footnotetext{
${ }^{8}$ If we substitute the numerical values obtained in 11] into the mass formula (3.24), we obtain an undesirable result in which inside of the square root in (3.24) becomes negative. The origin of this result gets back to the action (3.23). The sign of the coefficient of the terms quadratic in the gauge fields becomes negative, thus we cannot solve the Schrödinger problem with the unbounded potential. However, in the BSFT or the sigma-model approach, the coefficients $p_{1}=4 q^{4}, a, c$ are ambiguous and depend on the renormalization scheme [13]. Furthermore, since we have used the derivative-truncated action, the field redefinition does not close in our scheme. Hence it would be less sensible to substitute the values of the coefficient obtained in the BSFT. We thank S. Terashima for giving us a helpful comment on this point.
} 
Here $\epsilon$ is a regularization parameter, and we take $\epsilon \rightarrow 0$ later. Under the gauge condition (3.21), we obtain a classical solution

$$
T=q\left(x_{8}+i x_{9}\right), A_{\mu}=0
$$

where $2 q^{2}=\epsilon^{-1} \rightarrow \infty$. This solution is singular and thus avoiding Derrick's theorem. For investigation of the fluctuation spectra, we redefine the gauge fields in the same manner, $\hat{A}_{\mu} \equiv A_{\mu} \exp \left(-q^{2}\left(x_{8}^{2}+x_{9}^{2}\right) / 2 a\right)$. In the limit $\epsilon \rightarrow 0$, the action for the fluctuation is given as

$2 T_{\mathrm{D} 9} \int d^{10} x\left[q^{2}\left(x_{8}^{2}+x_{9}^{2}\right) \hat{A}_{\mu}^{2}+\frac{b}{2}\left\{\left(\frac{q^{4}}{a^{2}}\left(x_{8}^{2}+x_{9}^{2}\right)-\frac{2 q^{2}}{a}\right) \hat{A}_{\hat{\mu}}^{2}+\frac{q^{4}}{a^{2}}\left(x_{8}^{2} \hat{A}_{9}^{2}+x_{9}^{2} \hat{A}_{8}^{2}\right)+\frac{1}{2} \hat{F}_{\mu \nu}^{2}\right\}\right]$

where we extract up to second order of $\hat{A}_{\mu}$. Since we still have the cross terms again, we simply neglect the gauge fields $A_{8,9}$, then the mass squared for $A_{\hat{\mu}}$ is given by

$$
m_{A_{\hat{\mu}}^{(-)}}^{2}=(2 n+2) \sqrt{\frac{q^{4}}{a^{2}}+\frac{2 q^{2}}{b}}-\frac{2 q^{2}}{a}, \quad n \geq 0 .
$$

Thus the mass tower starts from the massive mode, which is consistent with the fact that the BPS D7-brane has a single massless gauge field coming from $A^{(+)}$in its effective theory.

\subsection{Universality of the fluctuation spectra}

We observed in Sec. 2.3 that, as for the kink solution, the results on the fluctuation in the two-derivative truncation are universal features found in a general action. In this subsection, following this success, we apply this generalization also to the vortex solution.

The general action we consider is the same as before, (2.30). If we include the gauge field $A^{(-)}$in the action, the number of the gauge-invariant terms is quite large. Thus to avoid this complication we again shall not consider this gauge field. We demand the solution to be the vortex configuration, $T=q\left(x_{8}+i x_{9}\right), A^{(+)}=0$. Then the equation of motion becomes the following condition for $f(X, Y)$ :

$$
\left[f-2 q^{2} \frac{\delta f}{\delta X}\right]_{X=2 q^{2}, Y=0}=0
$$

One of the preferable features of the generalized action (2.30) is that one can construct a D7-D5 bound state and (F, D7) bound state with correct ratio of the tensions by turning on the gauge fields on the worldvolume of the D7-brane vortex. See App. A for the details.

First, let us examine the tachyon fluctuations: $T=q\left(x_{8}+i x_{9}\right)+t_{1}(x)$. The quadratic action to which the fluctuation is subject is found as

$2 T_{\mathrm{D} 9} \frac{f\left(2 q^{2}, 0\right)}{2 q^{2}} \int d^{10} x\left[\left(\partial_{\hat{\mu}} \hat{t}_{1}\right)^{2}+C_{2} \hat{t}_{1}\left(-\partial_{8}^{2}-\frac{q^{2}}{a}+\frac{q^{4}}{a^{2}} x_{8}^{2}\right) \hat{t}_{1}+C_{3} \hat{t}_{1}\left(-\partial_{9}^{2}-\frac{q^{2}}{a}+\frac{q^{4}}{a^{2}} x_{9}^{2}\right) \hat{t}_{1}\right]$,

\footnotetext{
${ }^{9}$ We can say that this result is the same as what one obtains in $(3.24)$ with the two-derivative truncation limit $p_{1} \rightarrow 0$ (equivalently, $q \rightarrow \infty$ ) and $c=0$. In this sense, we have consistency.
} 
where

$$
C_{2} \equiv\left[1+\frac{4 q^{4}}{f}\left(2 \frac{\delta f}{\delta Y}+\frac{\delta^{2} f}{\delta X^{2}}\right)\right]_{X=2 q^{2}, Y=0}, \quad C_{3} \equiv\left[1+\frac{8 q^{4}}{f} \frac{\delta f}{\delta Y}\right]_{X=2 q^{2}, Y=0} .
$$

In obtaining the above action, we have already redefined $\hat{t}_{1} \equiv t_{1} \exp \left(-q^{2}\left(x_{8}^{2}+x_{9}^{2}\right) / 2 a\right)$ and used the condition (3.29). The mass squared is

$$
m_{t_{1}}^{2}=\frac{2 q^{2}}{a}\left(C_{2} n_{1}+C_{3} n_{2}\right) \quad \text { where } \quad n_{i} \geq 0, \quad i=1,2
$$

therefore the lowest mode is a massless mode. This is consistent with the previous analysis in Sec. 3.1.

Next, we study the gauge fluctuation $A^{(+)}$. The action for the fluctuation $A_{\mu}(x)$ is given by

$$
S=2 T_{\mathrm{D} 9} \frac{b f\left(2 q^{2}, 0\right)}{4} \int d^{10} x e^{-\frac{q^{2}}{a}\left(x_{8}^{2}+x_{9}^{2}\right)}\left(\left(F_{\hat{\mu} \hat{\nu}}^{(+)}\right)^{2}-2\left(F_{89}^{(+)}\right)^{2}\right) .
$$

Here, again, interesting action is obtained (recall eq. (2.39)). Above action tells us not only that the gauge potentials $A_{8,9}(x)$ are not mixed with the rest $A_{\hat{\mu}}$, but also that $A_{8,9}(x)$ have no kinetic term to run along the world volume of the D7-brane. Therefore they completely decouple from the physics on the D7-brane, except for the constant modes.

Thus, we showed the universality of the results obtained in Sec. 3.1 and 3.2. This general analysis shows that the field content on the vortex (= the BPS D7-brane) is properly given from the fluctuation spectra. The decent relation concerning the tachyon condensation from the D9D̄9 system to the BPS D7-brane in Type IIB string theory is examined at the level of the low-energy effective field theories.

\subsection{Derivation of the Dirac-Born-Infeld action}

Let us construct the effective action for the lowest fluctuation modes. The quadratic approximation gives only a trivial action of massless scalars and a gauge field. We would like to go further in order to see the resultant brane physics. In this subsection, we will find that the DBI action is derived in the following manner.

Let us focus on the massless modes $t_{1}$ and $t_{2}$. We can give them a meaning of the displacement fields, and this enables us to go beyond the quadratic approximation. In this subsection we turn on the constant gauge field strength $F_{\hat{\mu} \hat{\nu}}$ on the vortex worldvolume, and treat them as a background. We follow the procedure developed in [12]. At last we obtain the DBI action for the vortex D7-brane.

First, since the fields $t_{1,2}$ represent the transverse locations of the vortex in the $x_{8}-x_{9}$ plane, we can put them into the argument of the tachyon solution:

$$
T=T_{\text {sol }}\left(y_{8}, y_{9}\right), \quad y_{8} \equiv \frac{1}{\beta_{8}}\left(x_{8}+t_{1}\left(x_{\hat{\mu}}\right)\right), \quad y_{9} \equiv \frac{1}{\beta_{9}}\left(x_{9}+t_{2}\left(x_{\hat{\mu}}\right)\right) .
$$

Here $\beta$ 's are the boost factor obtained in the following manner. Let us consider $t_{i}(i=1,2)$ linear in $x_{\mu}$. These correspond to a special Lorentz transformation in the bulk, that is, a 
rotation of the vortex brane: $x \mapsto y \equiv \Lambda x$. Then, this rotation must preserve the metric on the brane as

$$
\left(\Lambda^{t}\right) G \Lambda=G
$$

The metric on the brane is the open string metric

$$
G^{\hat{\mu} \hat{\nu}}=\left(\frac{1}{1-b F^{2}}\right)^{\hat{\mu} \hat{\nu}}, \quad G^{88}=G^{99}=1,
$$

and $\Lambda$ is related to the displacement (3.33) as follows:

$$
\Lambda=\left(\begin{array}{cc|c}
1 / \beta_{8} & 0 & \partial_{\hat{\mu}} t_{1} / \beta_{8} \\
0 & 1 / \beta_{9} & \partial_{\hat{\mu}} t_{2} / \beta_{9} \\
\hline * & * & * \\
\vdots & \vdots & \vdots
\end{array}\right)
$$

We determine the unknown parameters in the Lorentz transformation matrix $\Lambda$ by substituting (3.36) into the condition (3.34). The result is

$$
\beta_{8}=\sqrt{1+G^{\hat{\mu} \hat{\nu}} \partial_{\hat{\mu}} t_{1} \partial_{\hat{\nu}} t_{1}}, \quad \beta_{9}=\sqrt{1+G^{\hat{\mu} \hat{\nu}} \partial_{\hat{\mu}} t_{2} \partial_{\hat{\nu}} t_{2}}, \quad G^{\hat{\mu} \hat{\nu}} \partial_{\hat{\mu}} t_{1} \partial_{\hat{\nu}} t_{2}=0 .
$$

The first and the second equation determine the boost factor. The third equation indicates that the Lorentz invariance is satisfied only if $\partial_{\hat{\mu}} t_{1}$ and $\partial_{\hat{\mu}} t_{2}$ are orthogonal to each other. This property is due to that our ansatz (3.36) for the Lorentz transformation does not include the rotation among $x_{8}$ and $x_{9}$.

Knowing the boost factor, we can proceed to obtain the effective action for $t_{1,2}$. Substituting (3.33) into the action (2.30) and performing the integration along the directions $x_{8,9}$ by changing the variables to the coordinates $y_{8,9}$, we obtain

$$
S=T_{\mathrm{D} 7} \int d^{8} x \beta_{8} \beta_{9} \sqrt{\operatorname{det}\left(1_{\hat{\mu} \hat{\nu}}+\sqrt{b} F_{\hat{\mu} \hat{\nu}}\right)} .
$$

Using the identity valid for arbitrary matrices [17]

$$
\operatorname{det}(M) \operatorname{det}\left(N+A M^{-1} A^{t}\right)=\operatorname{det}(N) \operatorname{det}\left(M+A^{t} N^{-1} A\right)=\operatorname{det}\left(\begin{array}{cc}
M & -A^{t} \\
A & N
\end{array}\right),
$$

it is possible to show that the action (3.38) is in fact the DBI action of the BPS D7-brane,

$$
S=T_{\mathrm{D} 7} \int d^{8} x \sqrt{\operatorname{det}\left(1_{\hat{\mu} \hat{\nu}}+\sqrt{b} F_{\hat{\mu} \hat{\nu}}+\partial_{\hat{\mu}} t_{1} \partial_{\hat{\nu}} t_{1}+\partial_{\hat{\mu}} t_{2} \partial_{\hat{\nu}} t_{2}\right)} .
$$

Here we have used the relations (3.37) and another orthogonality $\partial_{\hat{\mu}} t_{1}(F G)^{\hat{\mu} \hat{\nu}} \partial_{\hat{\nu}} t_{2}=0$.

Finally we have obtained the DBI action as an effective action of the vortex solution. Therefore, at the effective action level, we have shown that Sen's conjecture is correct, on the part concerning the vortex-type tachyon condensation, a D9D̄9 pair $\rightarrow$ a BPS D7-brane. 


\section{Conclusion and Discussion}

In this paper, we confirmed a part of the descent relations in Sen's conjecture, by using the low energy effective actions of a brane antibrane system. First, in Sec. 2, we analyzed the tachyon condensation from D9D̄9 system to non-BPS D8-brane using the effective action of the former. The action (2.12) allows a tachyon kink solution (2.18), and the fluctuation around them reproduces the precise low energy field content on a non-BPS D8-brane, 2.19) (2.24) (2.27). The lowest fluctuation modes of the tachyon and the gauge fields obey the action of the Minahan-Zwiebach model (2.28) which is an effective theory of the non-BPS D8-brane, thus we verified the descent relation (1) in Fig. 1 at the level of effective field theories.

Second, in Sec. 3, we carried out the similar analysis for the tachyon condensation (2) in Fig. 1, that is, from the D9D̄9 system to a BPS D7-brane, (3.14) (3.18) (3.28). We obtained correct low energy matter content on the D7-brane, and gave a positive evidence for that they are subject to the Dirac-Born-Infeld action (3.40).

Furthermore, in Sec. 2.3 and 3.3, we extended these analyses to include an arbitrary form of the action, and obtained the same results. Namely, a universality was found in the descent relations.

We should make comments about the assumptions we made in this paper and their validity. Throughout the paper we studied fluctuation spectra for field by field, however generically there are mixing terms even at the quadratic level. It seems very difficult to diagonalize the total system, and thus we neglected these cross terms naively, then the mass squared of the fluctuations are given correctly. One of the cross terms is between $t_{1}$ and $t_{2}$ in Sec. 3.1. One can show that the lowest modes of $t_{1}$ and $t_{2}$ obtained there satisfy also the total equations of motion of them with mixings, hence this mixing seems

irrelevant. However, we have no idea how to treat $A_{8,9}^{( \pm)}$and their mixing to $A_{\hat{\mu}}^{(-)}$in the vortex analysis in Sec. 3.2. The only salvation is that in the general treatment of Sec. 3.3, the mixing terms for $A^{(+)}$vanish generically. Thus we expect that this kind of decoupling may happen also for $A^{(-)}$if we properly include higher terms in $A^{(-)}$.

If we turn our eyes to the future possibility of the effective field theory approach of the $\mathrm{D} p \overline{\mathrm{D}} p$ system and the tachyon condensation, we find many directions. It is important to generalize our analysis to the non-Abelian case and check the fluctuation spectra around lower-dimensional D-branes via Atiyah-Bott-Shapiro (ABS) construction. It is interesting to construct the various brane configuration in the brane antibrane system using the effective theory, following the construction in [18, 19]. Some of the brane bound states are studied in the appendix.

The final discussion is devoted to the following question: what is the concrete form of the effective action which reproduces all the desirable properties appearing in the descent relations? As shown in Sec. 2.3 and 3.3, we can get universal results on the fluctuation spectra by allowing rather general action (2.30). Now, since we obtained all the mass spectra and various properties of the defects, we can demand that these properties should reproduce the appropriate values in string theory - the values concerning mass spacing, tensions and tachyon masses. Let us list our results and see how this requirement constrains the arbitrary function $f(X, Y)$ in the action. 
- Potential height. The potential height should be consistent with Sen's conjecture: the true vacuum of the tachyons can be interpreted as the annihilation of the two branes. Therefore,

$$
f(0,0)=1
$$

- Perturbative tachyon masses. In the original system of brane antibrane, we expand the potential and the function $f$, then the tachyon mass squared is given by

$$
\left[\frac{-f}{a}\left(\frac{\delta f}{\delta X}\right)^{-1}\right]_{X=Y=0}=\frac{-1}{2 \alpha^{\prime}}
$$

- Existence of the kink and vortex solutions. This requirement is (2.34) and (3.29), the existence of $q_{k}$ (for the kink) and $q_{v}$ (for the vortex) which satisfy

$$
\left[f-2 q_{k}^{2} \frac{\delta f}{\delta X}-4 q_{k}^{4} \frac{\delta f}{\delta Y}\right]_{X=q_{k}^{2}, Y=q_{k}^{4}}=0, \quad\left[f-2 q_{v}^{2} \frac{\delta f}{\delta X}\right]_{X=2 q_{v}^{2}, Y=0}=0 .
$$

- Tension of the defects. The kink solution (a non-BPS D8-brane) and the vortex solution (a BPS D7-brane) have their tensions $\mathcal{T}=\sqrt{2} T_{\mathrm{D} 8}$ and $T_{\mathrm{D} 7}$ respectively,

$$
2 T_{\mathrm{D} 9} f\left(q_{k}^{2}, q_{k}^{4}\right) \frac{\sqrt{a \pi}}{2 q_{k}}=\sqrt{2} T_{\mathrm{D} 8}, \quad 2 T_{\mathrm{D} 9} f\left(2 q_{v}^{2}, 0\right) \frac{a \pi}{4 q_{v}^{2}}=T_{\mathrm{D} 7} .
$$

- Mass spectrum of the fluctuation around the kink. From the mass spacing of $t_{1}$ and $t_{2}$, we have respectively

$$
\begin{aligned}
& {\left[\frac{4 q_{k}^{4}}{a f}\left(\frac{\delta f}{\delta X}+6 q_{k}^{2} \frac{\delta f}{\delta Y}+2 q_{k}^{2} \frac{\delta^{2} f}{\delta X^{2}}+8 q_{k}^{6} \frac{\delta^{2} f}{\delta Y^{2}}+8 q_{k}^{4} \frac{\delta^{2} f}{\delta X \delta Y} \cdot\right)\right]_{X=q_{k}^{2}, Y=q_{k}^{4}}=\frac{1}{\alpha^{\prime}},} \\
& {\left[\frac{f}{a}\left(2 \frac{\delta f}{\delta X}-\frac{f}{2 q_{k}^{2}}\right)^{-1}\right]_{X=q_{k}^{2}, Y=q_{k}^{4}}=\frac{1}{\alpha^{\prime}} .}
\end{aligned}
$$

- Mass spectrum of the fluctuation around the vortex. The spacing of the series of the mass squared is identified with the corresponding value in string theory,

$$
\frac{2 q_{v}^{2}}{a}\left[\left(1+\frac{8 q_{v}^{4}}{f} \frac{\delta f}{\delta Y}\right)\right]_{X=2 q_{v}^{2}, Y=0}=\frac{1}{\alpha^{\prime}}, \quad \frac{2 q_{v}^{2}}{a}\left[\frac{4 q_{v}^{4}}{f} \frac{\delta^{2} f}{\delta X^{2}}\right]_{X=2 q_{v}^{2}, Y=0}=\frac{n}{\alpha^{\prime}},
$$

for a certain positive integer $n$. Note that these two equations come from the two spacings of the mass squared in eq. (3.31).

- Consistency with the Minahan-Zwiebach model. If we simply put $T_{2}=0$ and replace $2 T_{\mathrm{D} 9}$ with $\sqrt{2} T_{\mathrm{D} 9}$ in the action (2.30), then we should have the non-BPS D9-brane action:

$$
S=\sqrt{2} T_{\mathrm{D} 9} \int d^{10} x e^{-T^{2} / a} \sqrt{\operatorname{det}(1+\sqrt{b} F)} f\left(X, Y=X^{2}\right)
$$


The tachyon field is real, and thus we have a relation $Y=X^{2}$. The analysis for this action was given by Minahan and Zwiebach [6, 7, 8], and that should be inherited to our action. Actually, the analysis for the kink in the above list gives sufficient conditions for the kink analysis of Minahan and Zwiebach.

Unfortunately, we observe that the above conditions are far less than what determines the form of the function $f(X, Y)$. In fact, the above relations only give particular relations only at given values of $X$ and $Y$, thus there is no information of functional degrees of freedom.

However, assuming that the parameters $q_{k}$ and $q_{v}$ diverge (which is actually realized in the BSFT), then we can extract some information on $f$ around the infinities of the arguments. The result is that we can choose various parameters in $f$ in such a way that all of the above conditions are satisfied.

\section{Acknowledgment}

K. H. would like to thank S. Hirano, T. Kuroki, T. Suyama, T. Takayanagi and S. Terashima for useful discussions and comments.

\section{A Bound States}

In this appendix, we construct various bound states of D-branes by generalizing the vortex solution (3.8) in the generalized action (2.30). This is a supporting evidence for the analysis using the general action (2.30).

In the action (2.30), the gauge field strength for $A^{(+)}$is coupled to the tachyon only through the metric $G^{\mu \nu}$ and the Born-Infeld-type front factor. Therefore, if the following three conditions are satisfied simultaneously, the total equations of motion are solved:

(i) The tachyon configuration is (3.8): $T=q\left(x_{8}+i x_{9}\right)$, and the function $f$ in the general action satisfies the condition (3.29) again.

(ii) The gauge fields transverse to the vortex worldvolume, $A_{8,9}$, vanish.

(iii) The other gauge fields $A_{\hat{\mu}}$ depend only on $x_{0}, \cdots, x_{7}$, and are subject to the BornInfeld equations of motion.

In particular, the equations of motion for the Born-Infeld theory can be solved of course by a constant field strength. This leads us to brane bound states. As an example, we turn

on only $F_{67}^{(+)}=$const. Then the resultant vortex solution is just a D7-D5 bound state in which the uniformly distributed D5-branes have their worldvolumes along $x_{0,1,2, \cdots, 5}$. The energy of the bound state can be calculated as

$$
E_{\mathrm{D} 7 \mathrm{D} 5}=\sqrt{\operatorname{det}(1+\sqrt{b} F)} 2 T_{\mathrm{D} 9} \frac{a}{q^{2}} f\left(2 q^{2}, 0\right) \int d^{8} x
$$


On the other hand, the D7-brane energy is

$$
E_{\mathrm{D} 7}=2 T_{\mathrm{D} 9} \frac{a}{q^{2}} f\left(2 q^{2}, 0\right) \int d^{8} x
$$

Thus we can obtain the correct result well-known in string theory,

$$
E_{\mathrm{D} 7 \mathrm{D} 5}=\sqrt{\operatorname{det}(1+\sqrt{b} F)} E_{\mathrm{D} 7} \text {. }
$$

This result is analogous to what was found in [12]. In addition, let us see how the Ramond-Raomnd charge of the lower-dimensional branes is realized in the CS coupling 10, 11

$$
S_{\mathrm{CS}}=2 T_{\mathrm{D} 9} \int C \wedge \operatorname{Tr} \sigma_{3} \exp \left(\begin{array}{cc}
F^{(1)}-|T|^{2} & d \bar{T} \\
d T & F^{(2)}-|T|^{2}
\end{array}\right) .
$$

Here we are sloppy about the numerical coefficients. Substituting the solution into eq. (A.4), we can obtain the D5-brane charge and the D7-brane charge:

$$
S_{\mathrm{CS}}=2 T_{\mathrm{D} 9} \int e^{-|T|^{2} / a} C^{(6)} \wedge\left(F_{67}^{(+)} d x_{6} \wedge d x_{7}\right) \wedge d T \wedge d \bar{T}+2 T_{\mathrm{D} 9} \int e^{-|T|^{2} / a} C^{(8)} \wedge d T \wedge d \bar{T}(A
$$

Another example is an $(\mathrm{F}, \mathrm{D} 7)$ bound state. Turning on an electric component $F_{07}=$ const, the energy of the Hamiltonian density is given as

$$
\mathcal{H}_{(\mathrm{F}, \mathrm{D} 7)}=\mathcal{L}-\Pi^{\mu} \dot{A}_{\mu}=\frac{1}{\sqrt{1-b F^{2}}} \mathcal{H}_{\mathrm{D} 7}
$$

Therefore we obtain

$$
E_{(\mathrm{F}, \mathrm{D} 7)}=\frac{1}{\sqrt{1-b F^{2}}} E_{\mathrm{D} 7},
$$

which is identical with the well-known result in string theory.

These phenomena intrinsically occur since the Born-Infeld action is factored out in the general action (2.30). Therefore, we can apply all the results in Born-Infeld dynamics in string theory, especially the various brane configurations realized in Born-Infeld theory.

\section{References}

[1] A. Sen, "Tachyon Condensation on the Brane Antibrane System", JHEP 9808 (1998) 012, hep-th/9805170; "Descent Relations Among Bosonic D-branes", Int. J. Mod. Phys. A14 (1999) 4061, hep-th/9902105; "Non-BPS States and Branes in String Theory", hep-th/9904207; "Universality of the Tachyon Potential", JHEP 9912 (1999) 027, hep-th/9911116. 
[2] E. Witten, "On Background Independent Open String Field Theory", Phys. Rev. D46 (1992) 5467, hep-th/9208027; "Some Computations in Background Independent Off-Shell String Theory", Phys. Rev. D47 (1993) 3405, hep-th/9210065;

K. Li and E. Witten, "Role of Short Distance Behavior in Off-Shell Open String Field Theory", Phys. Rev. D48 (1993) 853, hep-th/9303067;

S. Shatashvili, "Comment on The Background Independent Open String Theory", Phys. Lett. B311 (1993) 83, hep-th/9303143; "On The Problems with Background Independence in String Theory", hep-th/9311177.

[3] A. A. Gerasimov and S. L. Shatashvili, "On Exact Tachyon Potential in Open String Field Theory", JHEP 0010 (2000) 034, hep-th/0009103;

D. Kutasov, M. Marino and G. Moore, "Some Exact Results on Tachyon Condensation in String Field Theory", JHEP 0010 (2000) 045, hep-th/0009148;

D. Ghoshal and A. Sen, "Normalization of the Background Independent Open String Field Theory Action", JHEP 0011 (2000) 021, hep-th/0009191;

D. Kutasov, M. Marino and G. Moore, "Remarks on Tachyon Condensation in Superstring Field Theory", hep-th/0010108.

[4] M. Marino, "On the BV formulation of boundary superstring field theory", JHEP 0106 (2001) 059, hep-th/0103089;

V. Niarchos and N. Prezas, "Boundary Superstring Field Theory", Nucl. Phys. B619 (2001) 51, hep-th/0103102.

[5] O. Andreev, "Some Computations of Partition Functions and Tachyon Potentials in Background Independent Off-Shell String Theory", Nucl. Phys. B598 (2001) 151, hep-th/0010218.

[6] B. Zwiebach, "A Solvable Toy Model for Tachyon Condensation in String Field Thoery", JHEP 0009 (2000) 028, hep-th/0008227;

J. A. Minahan and B. Zwiebach, "Field Theory Models for Tachyon and Gauge Field String Dynamics", JHEP 0009 (2000) 029, hep-th/0008231.

[7] J. A. Minahan and B. Zwiebach, "Effective Tachyon Dynamics in Superstring Theory", JHEP 0103 (2001) 038, hep-th/0009246.

[8] J. A. Minahan and B. Zwiebach, "Gauge Fields and Fermions in Tachyon Effective Field Theories", JHEP 0102 (2001) 034, hep-th/0011226.

[9] S. Moriyama and S. Nakamura, "Descent Relation of Tachyon Condensation from Boundary String Field Theory", Phys. Lett. B506 (2001) 161, hep-th/0011002.

[10] P. Kraus and F. Larsen, "Boundary String Field Theory of the DD System", Phys. Rev. D63 (2001) 106004, hep-th/0012198. 
[11] T. Takayanagi, S. Terashima and T. Uesugi, "Brane-Antibrane Action from Boundary String Field Theory", JHEP 0103 (2001) 019, hep-th/0012210.

[12] G. Arutyunov, S. Frolov, S. Theisen and A. A. Tseytlin, "Tachyon condensation and universality of DBI action", JHEP 0102 (2001) 002, hep-th/0012080.

[13] A. A. Tseytlin, "Sigma model approach to string theory effective actions with tachyons", J.Math.Phys. 42 (2001) 2854, hep-th/0011033.

[14] H. Kawai and T. Kuroki, "Strings as Flux Tube and Deconfinement on Branes in Gauge Theories", Phys. Lett. B518 (2001) 294, hep-th/0106103;

G. 'tHooft, CERN preprint(1974);

J. Kogut and L. Susskind, "Vacuum polarization and the absense of free quarks in four dimensions", Phys. Rev. D9 (1974) 3501.

[15] G. Derrick, "Comments On Nonlinear Wave Equations As Models For Elementary Particles", J.Math.Phys. 5 (1964) 1252;

S. Coleman, "Aspects of Symmetry", Cambridge University Press, 1985.

[16] T. Suyama, "BPS Vortices in Brane-Antibrane Effective Theory", hep-th/0101002.

[17] G. W. Gibbons, "Born-Infeld particles and Dirichlet p-branes", Nucl. Phys. B514 (1998) 603, hep-th/9709027.

[18] K. Hashimoto and S. Hirano, "Brane Ending on Branes in A Tachyon Model", JHEP 0104 (2001) 003, hep-th/0102173.

[19] K. Hashimoto and S. Hirano, "Metamorphosis Of Tachyon Profile In Unstable D9Branes", Phys. Rev. D65 (2002) 026006, hep-th/0102174. 